\title{
Human Immunodeficiency Virus-1/Surface Glycoprotein 120 Induces Apoptosis through RNA-Activated Protein Kinase Signaling in Neurons
}

\author{
Mehrdad Alirezaei, ${ }^{1}$ Debbie D. Watry, ${ }^{1}$ Claudia F. Flynn, ${ }^{1}$ William B. Kiosses, ${ }^{2}$ Eliezer Masliah, ${ }^{3}$ Bryan R. G. Williams, ${ }^{4}$ \\ Marcus Kaul, ${ }^{5}$ Stuart A. Lipton, ${ }^{3,5}$ and Howard S. Fox ${ }^{1}$ \\ ${ }^{1}$ Molecular and Integrative Neurosciences Department and ${ }^{2}$ Core Microscopy Facility, The Scripps Research Institute, La Jolla, California 92037, \\ ${ }^{3}$ Department of Neurosciences, University of California at San Diego, La Jolla, California 92039, ${ }^{4}$ Monash Institute of Medical Research, Monash University, \\ Melbourne 3168, Victoria, Australia, and ${ }^{5}$ Center for Neuroscience, Stem Cell, and Aging Research, Burnham Institute for Medical Research, 10901 North \\ Torrey Pines Road, La Jolla, California 92037
}

Previous work has demonstrated that the surface glycoprotein (gp120) of human immunodeficiency virus-1 (HIV-1) can induce damage and apoptosis of neurons both in vitro and in vivo. In this report, we provide evidence that double-stranded RNA-activated protein kinase (PKR), a stress kinase, is involved in HIV/gp120-associated neurodegeneration. In cultures of mixed cortical cells, HIV/gp120 increased the protein level of PKR. Additionally, PKR was phosphorylated in neurons but not glia after exposure to gp120. The use of two independent pharmacological inhibitors of PKR activity abrogated neuronal cell death induced by gp120. Cortical neurons from PKR knock-out mice were significantly protected from neurotoxicity induced by gp120, further validating the pivotal proapoptotic function of PKR. gp120-induced phosphorylated PKR localized prominently to neuronal nuclei; PKR inhibition or the NMDA receptor antagonist MK-801 $[(+)$-5-methyl-10,11-dihydro-5H-dibenzo [a,d] cyclohepten-5,10-imine maleate] abrogated this effect. PKR inactivation also inhibited gp120-induced caspase-3 activation, consistent with its neuroprotective effect. Finally, brain tissue from individuals diagnosed with HIV-associated dementia (HAD), but not HIV infection alone, contained the activated form of PKR, which localized predominantly to neuronal nuclei. Together, these results identify PKR as a critical mediator of gp120 neurotoxicity, suggesting that activation of PKR contributes to the neuronal injury and cell death observed in HAD.

Key words: PKR; gp120; neurodegeneration; apoptosis; caspase-3; PACT

\section{Introduction}

Human immunodeficiency virus-1 (HIV-1) invades the CNS at an early phase of infection and induces progressive symptoms of motor and cognitive dysfunction as well as behavioral changes designated HIV-1-associated dementia (HAD) (McArthur et al., 2005). HAD is a neurodegenerative syndrome that results from damage to synapses and death of neurons in brain regions, such as the hippocampus, cerebral cortex, and basal ganglia (Mattson et al., 2005). Because neurons are not productively infected by HIV-1, the pathway of neuronal damage and apoptosis during this lentivirus infection is not completely understood (Kaul et al.,

\footnotetext{
Received Feb. 22, 2007; revised Aug. 16, 2007; accepted Aug. 20, 2007.

This work was supported in part by National Institutes of Health (NIH) Training Grant T32AG00252 and National Research Service Award F32NS048767 (M.A.), NIH Grants R01 EY09024, R01 NS046994, R01 NS047973, and P01 HD29587 (S.A.L.), R01 NS050621 (M.K.), R01 Al34039 (B.R.G.W.), R24MH059745 (supporting the California NeuroAIDS Tissue Network and E.M.), and R01 MH073490 and P30 MH062261 (H.S.F.). This is manuscript MIND \#18319 from The Scripps Research Institute. We thank Dr. E. Raz (University of California, San Diego, La Jolla, CA) for the PKR knock-out mice, Dr. T. Fujita (Institute for Virus Research, Kyoto University, Kyoto, Japan) for the PACT antibody, M. Zandonnati, L. Sionit, and J. Boyd for their technical assistance, Dr. J. Radford for suggestions for this manuscript, and Dr. C. Lanigan for statistical analysis support.

Correspondence should be addressed to Howard S. Fox, The Scripps Research Institute, 10550 North Torrey Pines Road, SP30-2030, La Jolla, CA 92037. E-mail: hsfox@scripps.edu.

DOI:10.1523/JNEUROSCI.2733-07.2007

Copyright $\odot 2007$ Society for Neuroscience $\quad$ 0270-6474/07/2711047-09\$15.00/0
}

2001). Nonetheless, evidence supports the complex role of such diverse factors as viral proteins, infected microglia/macrophages, and proinflammatory mediators, and their subsequent release of toxins, which leads to neuronal insult and HAD pathogenesis (Lipton and Gendelman, 1995; Kaul et al., 2001).

The HIV-1 surface glycoprotein 120 (HIV-1/gp120) is among the contributing viral factors that may induce neuronal damage and apoptosis through indirect and direct pathways in models studied in vitro and in vivo (Brenneman et al., 1988; Toggas et al., 1994; Bagetta et al., 1996; Meucci et al., 1998). As shown previously, coreceptors for gp120, in addition to CD4, include the chemokine receptors CXCR4 and CCR5, which are present on the surface of neurons and glial cells (Meucci et al., 1998; Kaul et al., 2001). Engagement of CXCR4 or CCR5 by gp120 activates intracellular signaling pathways mediating neuronal apoptosis (Lannuzel et al., 1995; Meucci et al., 1998; Kaul et al., 2007). Previous findings showing the presence of chemokine receptors on the surface of several CNS resident cell types raise the possibility that the association of gp120 with these receptors may contribute to the pathogenesis of neurological dysfunction.

Numerous studies have demonstrated that gp120 disrupts neuronal calcium homeostasis and causes mitochondria membrane disruption, cytochrome $c$ release, and subsequent activa- 
Table 1. Patient characteristics of cases evaluated for p-PKR/NeuN double immunohistochemistry in the frontal cortex

\begin{tabular}{|c|c|c|c|c|c|c|}
\hline Case & Age & Postmortem interval (h) & HIV status & Neuropathology & Diagnosis & p-PKR immunoreactivity \\
\hline 1 & 43 & 6 & $\mathrm{HIV}^{+}$ & HIVE & $H A D$ & +++ \\
\hline 2 & 31 & 8 & $\mathrm{HIV}^{+}$ & HIVE & $H A D$ & ++ \\
\hline 3 & 40 & 10 & $\mathrm{HIV}^{+}$ & HIVE & $H A D$ & + \\
\hline 4 & 43 & 12 & $\mathrm{HIV}^{+}$ & HIVE & $H A D$ & ++ \\
\hline 5 & 33 & 13 & $\mathrm{HIV}^{+}$ & HIVE & $H A D$ & + \\
\hline 6 & 34 & 4 & $\mathrm{HIV}^{+}$ & None & Normal & - \\
\hline 7 & 45 & 12 & $\mathrm{HIV}^{+}$ & None & Normal & $+1-$ \\
\hline 8 & 46 & 12 & $\mathrm{HIV}^{+}$ & None & Normal & $+1-$ \\
\hline 9 & 53 & 3 & $\mathrm{HIV}^{+}$ & None & Normal & $+1-$ \\
\hline 10 & 41 & 12 & $\mathrm{HIV}^{+}$ & None & Not available & - \\
\hline
\end{tabular}

The p-PKR immunoreactivity scores are given for $40 \times$ magnification fields in the frontal cortex.,$+++ \geq 20 \mathrm{NeuN}^{+} / \mathrm{p}_{\mathrm{PKR}}{ }^{+} ;++$, , between 10 and 20 NeuN-positive/p-PKR-positive cells;,+ between 1 and 10 NeuN-positive/p-PKRpositive cells; + /-, rare positive cells; - , no positive cells. HIVE, HIV encephalitis.

tion of caspases and endonucleases (Dreyer et al., 1990; Lannuzel et al., 1995; Mattson et al., 2005). HIV gp120 disrupts neuronal calcium homeostasis by perturbing calcium-regulating systems in the plasma membrane and endoplasmic reticulum (ER) (Medina et al., 1999; Haughey and Mattson, 2002). Additionally, gp120 activates macrophages and microglia, which in turn initiate a cascade of glutamate-related hyperactivation of NMDA receptors on neurons. Such activation of NMDA receptors is also known to contribute to ER stress and release of $\mathrm{Ca}^{2+}$ (Lipton et al., 1991; Lei et al., 1992). ER stress appears to act early in the cell death process (Williams, 1999). Thus, ER stress is commonly associated with many neurodegenerative disorders (Bando et al., 2005).

The depletion of ER $\mathrm{Ca}^{2+}$ stores has been found to signal the activation of double-stranded (ds) RNA-activated protein kinase (PKR) in the SH-SY5Y neuronal cell line (Garcia et al., 2006; Shimazawa and Hara, 2006). Furthermore, thapsigargin, which induces the depletion of intracellular $\mathrm{Ca}^{2+}$ stores, leads to PKR activation (Prostko et al., 1995) and promotes neuronal apoptosis (Shimazawa and Hara, 2006; Uehara et al., 2006). PKR is a serine/threonine protein kinase that is ubiquitously expressed in all mammalian cells at basal levels and can be induced during treatment with interferon (IFN) or other agents (Balachandran et al., 1998; Bonnet et al., 2000; Garcia et al., 2006). PKR plays an important role in antiviral defense by IFN (Balachandran et al., $1998)$ and in nuclear factor $\kappa \mathrm{B}(\mathrm{NF}-\kappa \mathrm{B})$ activation (Bonnet et al., 2000, 2006; Garcia et al., 2006). This kinase also functions as a signal transducer in the proinflammatory response to multiple agents (Williams, 1999; Garcia et al., 2006). Furthermore, PKR has been implicated in the induction of stress responses and apoptosis (Yeung et al., 1996; Srivastava et al., 1998; Williams, 1999; Garcia et al., 2006). PKR has also been detected in the nuclei of human and murine cells (Jagus et al., 1999; Onuki et al., 2004). Interestingly, its translocation to the nucleus in a human neuronal cell line has been associated with stress-induced apoptosis (Onuki et al., 2004; Garcia et al., 2006).

The notion that PKR acts solely as a sensor for viral infection has been challenged by the discovery of a virus-independent activator of PKR, a cellular protein called PACT (PKR-activating protein) (Patel and Sen, 1998). PACT binds PKR and activates PKR through a dsRNA-independent mechanism (Peters et al., 2001). The binding of PACT to PKR promotes conformational changes that allow activation of PKR via its phosphorylation. Activated (phospho)-PKR (p-PKR) then induces the downstream signaling pathways, leading to the activation of NF- $\kappa \mathrm{B}$. PACT has been proposed as an intermediate in PKR-induced apoptosis (Gilbert et al., 2002). In fact, overexpression of PACT causes apoptosis in wild-type mouse embryonic fibroblasts, whereas transfection into fibroblasts lacking PKR does not. As expected, when PKR is cotransfected with PACT into the same PKR-deficient cells, the apoptotic response is restored (Peters et al., 2001).

Evidence is now accumulating that proinflammatory PKR is involved in neurodegeneration, and the activated form of PKR has been detected in the brains of patients with Alzheimer's disease (Chang et al., 2002a,b; Peel and Bredesen, 2003; Suen et al., 2003; Onuki et al., 2004; Garcia et al., 2006), Huntington's disease (Peel et al., 2001; Bando et al., 2005), Parkinson's disease (Bando et al., 2005), and amyotrophic lateral sclerosis (Hu et al., 2003; Garcia et al., 2006). In the present investigation, our goal was to determine whether activation of proapoptotic PKR participates in HIV-1/gp 120-associated neurodegeneration in a mixed rat cortical cell culture and validate our findings in autopsied brain slices from patients with HAD.

\section{Materials and Methods}

PKR knock-out mice. $\mathrm{PKR}^{0 / 0}$ mice (Yang et al., 1995) were backcrossed onto the C57BL/6J genetic background in Dr. E. Raz's laboratory at University of California, San Diego (La Jolla, CA) and transferred to The Scripps Research Institute vivarium. Wild-type C57BL/6J mice were purchased from The Jackson Laboratory (Bar Harbor, ME).

Preparation and treatment of primary rat or mouse cerebrocortical cultures. Primary neurons were cultured as described previously (Kaul and Lipton, 1999). In brief, the cerebrocortices of Sprague Dawley rat embryos at day 17 of gestation or $\mathrm{PKR}^{0 / 0}$ and wild-type mouse embryos at day 15 of gestation were digested and plated on poly-L-lysine (30-70 $\mathrm{kDa}$; Sigma, St. Louis, MO) coated glass coverslips at a density of $1 \times 10^{6}$ cells $/ \mathrm{ml}$ in high-glucose DMEM supplemented with $10 \%$ bovine calf serum, $25 \mathrm{mg} / \mathrm{ml}$ streptomycin, and $2 \mathrm{~mm}$ glutamine.

Cells were used for experiments after $21 \mathrm{~d}$ in culture. These cultures contained neurons, astrocytes, and microglia/macrophages, as determined with specific immunolabeling. Cultures were transferred into Earl's balanced salt solution (EBSS) containing $1.8 \mathrm{mM} \mathrm{Ca}^{2+}$ plus $5 \mu \mathrm{M}$ glycine and pretreated, when indicated, for $30 \mathrm{~min}$ with 2-aminopurine (2-AP) (Biomol, Plymouth Meeting, PA) or PKRi (Calbiochem, San Diego, CA) and then exposed to gp120 IIIb strain (Immunodiagnostic, Woburn, MA) for $24 \mathrm{~h}$. Immunostaining and Western blotting were performed for additional analysis.

Human tissues. The California NeuroAIDS Tissue Network provided clinical information and postmortem tissue from HIV-infected patients, obtained under Institutional Review Board approval from University of California, San Diego. Samples from 10 HIV-infected individuals were studied, five with the clinical diagnosis of HAD and five who had AIDS but no dementia. For additional patient details, please refer to Table 1.

Western blot analysis. Cells were lysed in buffer $(20 \mathrm{~mm}$ Tris-Cl, 150 mм NaCl, 1 mм EDTA, 1 mm EGTA, 1\% Triton X-100, 2.5 mm sodium pyrophosphate, $1 \mathrm{~mm}$ glycerol phosphate, and $1 \mathrm{~mm} \mathrm{Na}_{3} \mathrm{VO}_{4}$, with complete protease inhibitors). Protein concentration was determined with the bicinchoninic acid method, using bovine serum albumin as standard. 
Proteins were separated on NuPAGE $4-12 \%$ Bis-Tris acrylamide gradient gels (Invitrogen, Carlsbad, CA) and transferred onto Hybond-ECL nitrocellulose membrane (GE Healthcare, Little Chalfont, UK). Immunoblots were incubated in blocking solution for $1 \mathrm{~h}$ and then overnight at $4^{\circ} \mathrm{C}$ with primary mouse monoclonal anti-PKR antibody (1:250; BD Biosciences, San Jose, CA) or rabbit polyclonal anti-PACT antibody (1: 500; generously provided by Dr. T. Fujita, Institute for Virus Research, Kyoto University, Kyoto, Japan) (Iwamura et al., 2001). Next, immunoblots were washed, incubated with secondary anti-rabbit HRP antibodies (1:2000; GE Healthcare) for $1 \mathrm{~h}$ at room temperature, washed again, and developed using the chemiluminescence method (ECL plus kit; GE Healthcare). The blots were then stripped and reprobed with anti- $\alpha$ tubulin antibody (1:5000; Sigma) to assess the total protein load. Films were scanned, and the densitometry analysis was performed by using NIH ImageJ software (version 1.36b).

Double immunocytochemistry. After different treatments, cells were fixed for 25 min with ice-cold $4 \%$ paraformaldehyde (PFA) at $4^{\circ} \mathrm{C}$ and washed three times in PBS, at room temperature. PFA-fixed cells were permeabilized by using $0.2 \%$ Tween $20 / \mathrm{PBS}$, and nonspecific binding sites were blocked by incubation for $1 \mathrm{~h}$ with a $10 \%$ solution of heatinactivated goat serum in $0.2 \%$ Tween $20 / \mathrm{PBS}$. To specifically stain neurons, astrocytes, and microglia, cells were then incubated for $1 \mathrm{~h}$ at room temperature with dilutions of anti-microtubule associated protein-2 (MAP-2) (Sigma) at 1:500, glial fibrillary acidic protein (GFAP) (Sigma) at 1:400, and anti-OX-42 (Serotec, Raleigh, NC) at 1:1000. After several washes, cells were incubated for $1 \mathrm{~h}$ with different antibodies directed against PKR (1:250; Santa Cruz Biotechnology, Santa Cruz, CA) or PACT (1:500) (Iwamura et al., 2001). Cells were then incubated with secondary polyclonal antibodies, Alexa Fluor 488-conjugated goat anti-rabbit (Invitrogen) at 1:500 and Rhodamine Red-X-conjugated goat anti-mouse $\operatorname{IgG}$ (Invitrogen) at 1:500 for $1 \mathrm{~h}$ at room temperature. Cells were counterstained with the fluorescent nuclear stain 4'6-diamidino-2phenylindole (DAPI) (Sigma) at $1 \mu \mathrm{g} / \mathrm{ml}$, air dried, and mounted on glass microscope slides in Vectashield mounting media (Vector Laboratories, Peterborough, UK). Images from cells were visualized and acquired using a Zeiss (Oberkochen, Germany) Axiovert 200 inverted microscope at $40 \times$ magnification and captured by using the Zeiss Axiocam HRC associated with Zeiss Axiovision 2.0.5 software package. Controls for the immunostaining experiments were performed by omitting, sequentially, each of primary antibodies to obtain negative controls for cross-reactivity or spurious secondary antibody binding.

Confocal microscopy and image analysis. For the analysis of PKR and p-PKR by confocal microscopy, cells were coimmunostained with the nuclear marker neuronal-specific nuclear protein (NeuN) (1:500; Chemicon, Temecula, CA) and PKR (1:250; Santa Cruz Biotechnology, Santa Cruz, CA) or p-PKR (1:100; Epitomics, Burlingame, CA). All images were acquired using a Rainbow Radiance 2100 Laser Scanning Confocal system attached to a Nikon (Tokyo, Japan) TE2000-U inverted microscope (Bio-Rad, Hercules, CA). Optical image slices $(0.2 \mu \mathrm{m}$ interval step slices) were acquired using Laser Sharp 2000 software and then imported and further analyzed for detailed quantitative colocalization using three independent software packages: Laser Sharp (Bio-Rad), LSM examiner (Zeiss), and NIH Image J (http://rsb.info.nih.gov/ij). For each triple-labeled image (NeuN-red, PKR-green or p-PKR-green, and DAPI), NeuN-positive neuronal cells were first outlined and then were used to assess colocalization. Colocalization between two fluorescently labeled reagents (PKR-green or p-PKR-green and DAPI) was quantified by first obtaining the signal intensity threshold range of real over background signal using controls. The same approach was used for nuclear and cytoplasmic colocalization of PKR/MAP-2 and PKR/DAPI. The average real fluorescence signal threshold range was then used to define the spatial position, thus area, in the cell that the two independent fluorescent signals occupy, and this information was used to calculate the correlation coefficients ( $M$ values refers to ratio of spatial overlap as defined by the fluorescent signal) for at least 225 cells over four separate experiments. The correlation coefficient obtained were converted to a percentage representing the percent of 100 of the total area, defined by the spatial location of the fluorescently labeled PKR signal, that resides in the nucleus over the various conditions.
Double immunohistochemistry. Briefly, $5 \mu \mathrm{m}$ sections of Formalinfixed, paraffin-embedded tissue were mounted on charged slides were deparaffinized and hydrated. The antigenic sites were unmasked with a $0.01 \mathrm{~m}$ citrate buffer, $\mathrm{pH} 6$, in a steamer. Endogenous peroxidase was blocked by a 30 min treatment with $3 \% \mathrm{H}_{2} \mathrm{O}_{2}$ in $10 \%$ methanol. Nonspecific binding was blocked with $10 \%$ goat serum $/ 0.1 \%$ Triton $\mathrm{X}-100$ in PBS. Slides were incubated overnight with the primary antibody antiPKR $\mathrm{p}\left[\mathrm{T}^{451}\right]$ (1:1000; Invitrogen) and anti-NeuN (1:500; Chemicon) in $\mathrm{PBS} / 10 \%$ goat serum block solution, under low agitation, at $4^{\circ} \mathrm{C}$. Slides were incubated for $1 \mathrm{~h}$ with Alexa Fluor 488 (green) and Rhodamine Red-X (red) and then were counterstained with DAPI and mounted. Images from five fields per section $(20 \times$ magnification) were visualized and acquired as described above. Negative controls were performed using non-immune serum and absorption with antigen as described in Results.

Assessment of neuronal apoptosis. Cells were stained with MAP-2 and counterstained with DAPI. MAP-2 and DAPI-labeled nuclei were counted based on their nuclear morphology (apoptotic $=$ condensed nuclei) in five randomly selected fields using a $40 \times$ objective using a Zeiss Axiovert 200 microscope, and neuronal apoptosis results were expressed on a graph by using Prism software (GraphPad Software, San Diego, CA).

Caspase-3 activity. Cultures were transferred into EBSS containing 1.8 mм $\mathrm{Ca}^{2+}, 5 \mathrm{~mm}$ glycine, pretreated with PKRi or caspase- 3 inhibitor [Ac-IETD-CHO ( $N$-Acetyl-Ile-Glu-Thr-Asp-aldehyde)] for $30 \mathrm{~min}$, and then exposed to gp120 for $24 \mathrm{~h}$ unless mentioned otherwise in the figure legends. NMDA at $100 \mu \mathrm{M}$ was added for only $10 \mathrm{~min}$ and was used as the positive control for caspase-3 activation. We then performed the assay with a Promega (Madison, WI) kit according to the protocol and recommendation of the manufacturer. Caspase- 3 activity was determined using a Multilabel Counter 1420 Victor (PerkinElmer, Boston, MA). Relative fluorescence units were normalized by considering the average of control fluorescence data.

Statistical analysis. Statistical analysis was performed using GraphPad Software Instat. All experiments were repeated at least three times, unless otherwise stated. Data were analyzed by an ANOVA (one-way) followed by post hoc Tukey's test to make all possible comparisons. Data were expressed as means \pm SEM, and significance was assessed at $p<0.05$.

\section{Results}

\section{PACT is expressed in neurons}

Intracellular PKR is often activated by virally derived dsRNA. Although neurons are not infected by HIV, it is known that PKR can be activated in a dsRNA-independent manner. The endogenous PKR activator is PACT. To determine whether PACT was present and induced in neurons, immunostaining was performed, and protein extracts were prepared from cortical cell cultures that had been subjected to gp120 in a time course manner. PACT protein expression was present, but its level was not significantly changed after exposure to gp120 (data not shown). Staining with anti-PACT antibody illustrated its expression in neurons (Fig. 1a,b).

\section{PKR induction in cultures exposed to gp 120}

Because the level of PKR expression generally increases before its activation (Williams, 1999; Bonnet et al., 2000; Garcia et al., 2006), we first examined whether PKR is induced in rat mixed cortical cell cultures exposed to gp 120. Western blot analysis (Fig. $1 c, d)$ revealed that gp120 exposure resulted in an increase in PKR protein levels, with a maximum of a $3.2 \pm 0.2$-fold increase after 9 h (Fig. 1d).

Glial cells are required for gp120 to execute its neurotoxic effects on neurons in our mixed cortical culture model (Kaul and Lipton, 1999). To determine whether PKR induction occurred in glia or neurons, double-immunofluorescence analysis was performed (Fig. 1e-j). The results revealed that $>95 \%$ of the cells in 
which total PKR (green fluorescence) was upregulated after exposure to gp120 for $9 \mathrm{~h}$ were neurons (MAP-2 positive) (Fig. $1 f$, white arrows). Of all MAP-2-positive neurons, $36 \pm 1.1 \%$ showed upregulated PKR, but PKR was not induced in some neurons (Fig. $1 f$, pink arrows). In these mixed cultures, other than neurons, few other cell types expressed PKR (Fig. $1 f$, blue arrow). Double-immunofluorescence analysis indicated that these were astrocytes (GFAP positive); only a small percentage ( $2 \pm$ $0.9 \%$ ) of the GFAP-positive astrocytes expressed PKR, and, in general, they expressed low levels of PKR (Fig. 1h, pink arrows) compared with that found in neurons (the non-GFAP reactive cells) (Fig. $1 h$, blue arrows). Next, to determine whether PKR was upregulated in microglia cells, we double stained with antibodies directed against microglia (OX-42) and total PKR in mixed cortical cell cultures. However, these microglial cells did not express PKR in response to gp120 (Fig. $1 j$, pink arrows). All these outcomes (Fig. 1e-j) demonstrated that the upregulation of PKR, detected by Western blot analysis (Fig. $1 c, d$ ), appeared predominantly in neurons and suggest that PKR, as a stress kinase, may be implicated in the neuronal response to gp120-treated neuroglial cultures.

\section{PKR activity is a prominent factor in gp120 neurotoxicity}

From previous studies, it is known that exposure to gp120 induces the degeneration of rat neurons. In accord, our results demonstrated that gp120 (200 pM) effectively provoked apoptosis of these cells in culture $(29.6 \pm 1.2 \%$ compared with the basal level of $8.6 \pm 0.6 \%$ ) (Fig. $2 a$ ). To examine our hypothesis that proapoptotic p-PKR plays a role in this context, we evaluated an inhibitor of PKR, 2-AP, which shows specificity for PKR at low millimolar levels (Huang et al., 2003). When added to the cultures, $1 \mathrm{~mm}$ 2-AP significantly abrogated the neuronal death induced by gp120 (12.1 $\pm 0.8 \%)$. Next, we tested a newly described, higher-affinity specific inhibitor of PKR [8-(imidazol-4ylmethylene)-6 $\mathrm{H}$-azolidino[5,4-g]benzothiazol-7-one], which has an imidazole structure (referred to here as PKRi) and has been shown to inhibit RNA-induced PKR autophosphorylation (Jammi et al., 2003; Shimazawa and Hara, 2006). In initial control experiments, concentrations of 2, 20, and $200 \mathrm{nM}$ PKRi did not cause neurotoxicity themselves, but, at concentrations $\geq 2 \times 10^{3}$ $\mathrm{nM}$, toxicity was evident (Fig. $2 b$ ). Thus, we studied the effect of 2-200 nM PKRi on gp120-induced neurotoxicity in cortical cell cultures. We found that 2 and $20 \mathrm{~nm}$ PKRi (Fig. 2c) partially protected neurons (reducing gp120 neurotoxicity from $32.1 \pm$ $2.1 \%$ to $23.6 \pm 0.9$ and $12.3 \pm 0.8 \%$, respectively), whereas 200 nM PKRi more completely protected against gp 120 neurotoxicity ( $9 \pm 0.8 \%$ toxicity compared with the basal level of $6.3 \pm 0.6 \%$ ). The reduction in toxicity at all concentrations of PKRi was significant. These results demonstrated that this type of cell death could be prevented by inactivating PKR.
Because pharmacological inhibitors may not be perfectly selective for their targets, we next sought a molecular approach to determine whether PKR contributed to gp120-induced neuronal damage. We therefore tested whether cortical cultures deficient in PKR could avoid apoptosis signaling triggered by gp120. Thus, we compared the neurotoxicity induced by gp120 in cortical cell cultures from PKR knock-out mice $\left(\mathrm{PKR}^{0 / 0}\right)$ with that obtained from wild-type mice (Yeung et al., 1996). $\mathrm{PKR}^{0 / 0}$ cortical cultures exposed to gp120 manifest threefold lower neurotoxicity (Fig. $2 d$, $10.9 \pm 0.6 \%$ vs basal level $7.1 \pm 0.7 \%$ ) than the similarly gp 120 exposed wild-type cultures (Fig. $2 d, 33.3 \pm 2.2 \%$ vs basal level $7 \pm 0.5 \%)$. For both genotypes, the use of heat-inactivated gp120 did not result in increased neurotoxicity. Together with the above results using inhibitors, these data are therefore consistent with PKR playing a central role in gp120-induced neuronal apoptosis.

\section{gp120 induces the activation of PKR and its} nuclear translocation

In the brains of individuals with Huntington's disease, $\mathrm{p}-\mathrm{PKR}$ is located predominantly in neuronal nuclei (Peel et al., 2001). Moreover, it has been reported that an agent inducing ER stressmediated apoptosis (tunicamycin) is able to induce PKR translocation from cytoplasm to nucleus in a neuronal cell line (Onuki et al., 2004). To assess whether gp120 has a similar effect, we first tested whether exposure to gp120 induces PKR nuclear translo- 

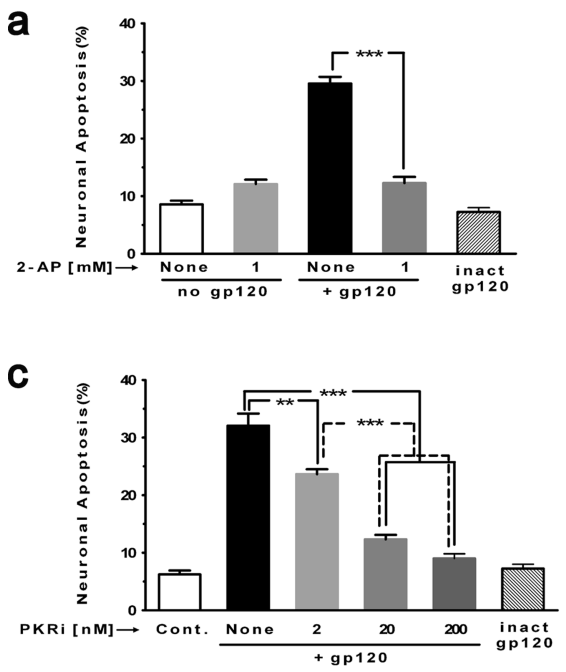
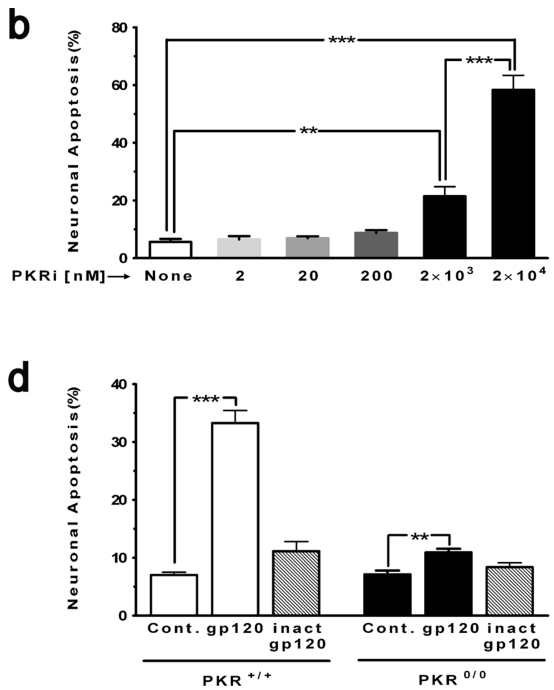

Figure 2. Prominent role of PKR in gp120 neurotoxicity. When indicated, cortical cell cultures were pretreated with an inhibitor of PKR (30 min) and then exposed to gp120 (200 pM). Immunostaining was performed with an antibody directed against MAP-2 to identify neurons and DAPI for nuclear staining. Apoptotic nuclei were counted from different regions on coverslips. $\boldsymbol{a}, 2-\mathrm{AP}$ (1 $\mathrm{mm}$ ) completely protected neurons against toxicity induced by gp120. 2-AP by itself was not neurotoxic. ${ }^{* * *} p<0.001$ for $n=4$ experiments. $\boldsymbol{b}$, PKRi was nontoxic at 2, 20, and 200 nm but became toxic at in the micromolar range. ${ }^{* * *} p<0.001,{ }^{* *} p<0.01$ for $n=3$ experiments. c, PKRi (2-200 nm) abrogated gp120 neurotoxicity in a dose-dependent manner. Heat-inactivated gp120 (inact gp120) served as a control (Cont.) and was not neurotoxic. ${ }^{* *} p<0.001,{ }^{* *} p<0.01$ for $n=4$ experiments. $\boldsymbol{d}$, Only minimal neurotoxicity occurs in cortical cultures from PKR knock-out mice versus wild type (the phenotypic appearance of PKR ${ }^{0 / 0}$ cultures resembled that of wild-type mouse and rat cortical cell cultures; data not shown). Heat-inactivated (inact) gp120 was used as a negative control for the neurotoxicity experiments. ${ }^{* *} p<0.001,{ }^{* *} p<0.01$ for $n=4$ experiments. All values are mean \pm SEM.

cation. Indeed, the data demonstrate that, in neurons exposed to gp120 for $24 \mathrm{~h}$, the PKR cytoplasmic/nucleus ratio was decreased by $79.9 \pm 11.9 \%$, indicating the increased nuclear localization of PKR (Fig. 3a). Next, we quantified the gp120-induced change in the localization of PKR in neuronal nuclei. Data obtained from confocal imaging illustrated that total PKR is increased by $28.9 \pm$ $1.2 \%$ in neuronal nuclei after exposure to gp120 for $24 \mathrm{~h}$ (Fig. $3 b, c)$.

Because we found that the activity of PKR was necessary for gp120-induced neuronal death, we then examined whether the active form of PKR was translocated to neuronal nuclei after exposure to gp120. In addition, because NMDA receptor activation is a known mediator of HIV-induced neurotoxicity, we tested whether the gp120-induced PKR translocation may be prevented by using an antagonist of the NMDA receptor (MK-801 [(+)-5-methyl-10,11-dihydro-5H-dibenzo [a,d] cyclohepten5,10-imine maleate]). Confocal analysis revealed that $\mathrm{p}-\mathrm{PKR}$ was significantly more abundant in neurons exposed to gp120 for $24 \mathrm{~h}$ (Fig. $3 d, e)$. As expected, the increase of p-PKR in neuronal nuclei was blocked by the presence of PKRi at $200 \mathrm{nM}$. Interestingly, MK-801 at $10 \mu \mathrm{M}$ also blocked the nuclear increase of p-PKR (Fig. 3e), indicating that NMDA signaling is an important pathway in gp120-inducing neuronal death and upstream of PKR signaling pathway.

PKRi inhibits caspase- 3 activity induced by gp 120

Both PKR- and gp120-induced apoptosis are linked to caspase-3 activity (Garden et al., 2002; Suen et al., 2003; Garcia et al., 2006; Scheuner et al., 2006). To better verify the potential role of PKR in gp120-induced caspase-3 activation in neurons, we examined whether inactivation of PKR has an inhibitory effect on caspase-3 activation. For that purpose, we measured caspase-3 activity in rat cerebrocortical cultures pretreated with PKRi and then ex-

posed to gp120. The results indicated that the increased level of caspase- 3 activity caused by gp120 was significantly $(p<$ 0.001 ) reduced (statistically indistinguishable from control levels) in the presence of 200 nM PKRi (Fig. 4). These results are consistent with the notion that the inactivation of PKR inhibits caspase- 3 activity induced by gp120 and that PKR acts as an intracellular mediator for gp120, eliciting caspase activation and subsequent induction of neuronal apoptosis.

Furthermore, because the caspase- 3 activity was measured in mixed cortical cultures, we then analyzed the presence of activated caspase- 3 in neurons using confocal imaging, revealing that the activated form of caspase- 3 is localized within MAP-2-positive cells after exposure to gp120 (data not shown), as has been reported previously (Garden et al., 2002). Our experiments have now expanded these studies to show the dependence on PKR, because the increased level of activated caspase- 3 induced in neurons exposed to gp120 is reduced significantly in the presence of PKRi (Fig. 4).

\section{p-PKR in brains of HAD patients}

To correlate the results of our experimental model with HAD, we performed immunohistochemical analysis to detect p-PKR in samples from human brains. Indeed, p-PKR was completely absent (Fig. $5 a-c$ ) from the brain tissues of patients who were HIV positive but did not have HAD, but in sections of HAD-positive patients (Fig. $5 d-f$ ) p-PKR colocalized with the neuronal marker NeuN. Substitution of the primary antiserum with normal goat serum (data not shown) or incubation of the p-PKR antibody with p-PKR peptide (pp-PKR) (Fig. $5 g-i)$ obliterated the staining in tissues from the patients with HAD. In addition to the data obtained from hippocampus (Fig. $5 a-i)$, similar results were obtained on brain sections from both cortex and basal ganglia (data not shown). This finding confirms the presence of activated PKR in the brains of individuals with HAD and supports the possible contribution of PKR to HIV neuropathology.

\section{Discussion}

In the current study, we propose a novel mechanism implicating PKR activation in HIV-associated neurodegeneration, a hallmark of HAD. We demonstrate for the first time that PKR activity is turned on during neuronal apoptosis. Moreover, the neurotoxicity of gp120 is significantly reduced in cultures of cortical cells deficient in PKR or completely when treated with PKR-specific inhibitors. It is possible that PKR is activated downstream of gp120 signaling directly on neurons or, alternatively, via glial toxins acting on neurons, e.g., via NMDA receptor activation (Fig. 6). Previous data support the NMDA pathway as the predominant one in HIV/gp120-induced neurotoxicity (Lipton et al., 1991; Lei et al., 1992; Kaul and Lipton, 1999). Furthermore, our results (Fig. 3e) determined that the NMDA receptor is involved in the neurotoxic PKR signaling. Importantly, our in vitro studies correlate with the increased p-PKR found in the brains of 


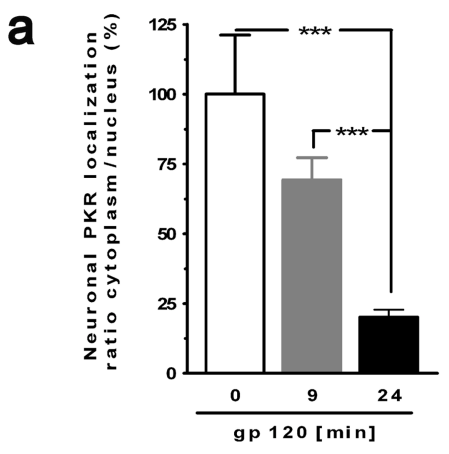

C

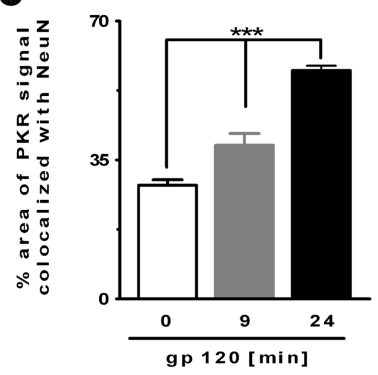

e

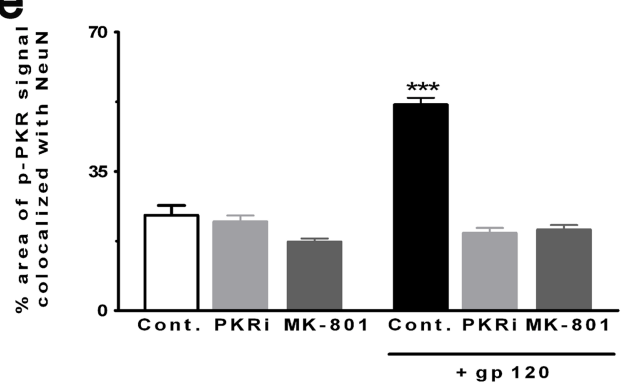

b

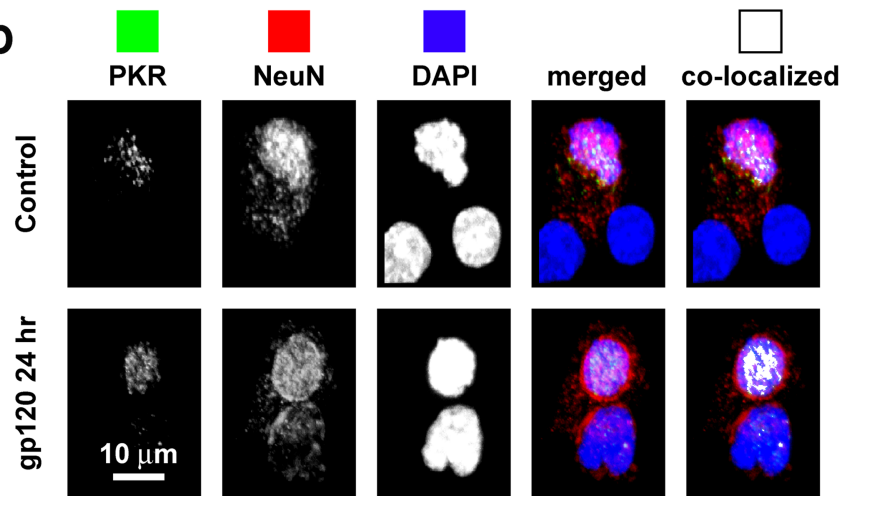

d

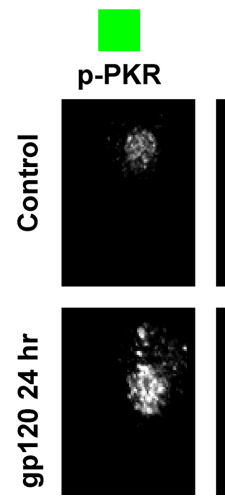

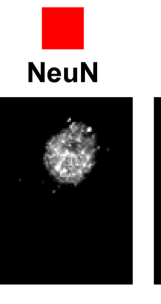

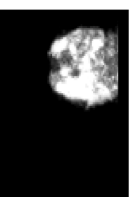

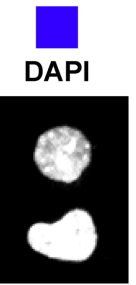

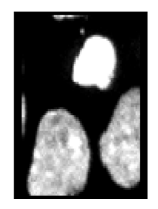

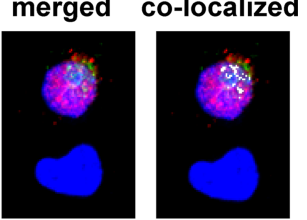
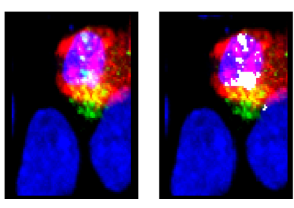

Figure 3. PKR and p-PKR translocation to nucleus in neurons exposed to gp120. $\boldsymbol{a}$, PKR colocalization with MAP-2 (cytoplasmic) and DAPI (nuclear) illustrates the PKR translocation to nuclei in cultures exposed to gp120. ${ }^{* * *} p<0.001$ for $n=4$ experiments. $\boldsymbol{b}$, Images acquired using confocal microscopy of neurons (NeuN positive; red) exposed to gp 120 for $24 \mathrm{~h}$ with PKR (green) and counterstained with DAPI. Colocalized PKR with NeuN is shown in white in the far right panels. c, PKR colocalization with NeuN revealed an increase of PKR in nuclei after exposure to gp $120 \mathrm{for} 24 \mathrm{~h}$. ${ }^{* * *} p<0.001$ for $n=4$ experiments. $\boldsymbol{d}$, Images acquired using confocal microscopy of neurons (NeuN positive, red) exposed to gp120 for $24 \mathrm{~h}$ with p-PKR (green) and counterstained with DAPI. Colocalized p-PKR with NeuN is shown in white in the far right panels. $\boldsymbol{e}$, Increased colocalization of p-PKR and NeuN in neurons exposed to gp 120 after $24 \mathrm{~h}$, which is abrogated in the presence of PKRi or MK-801. ${ }^{* * *} p<0.001$ for $n=4$ experiments. Cont., Control. All values are mean \pm SEM.

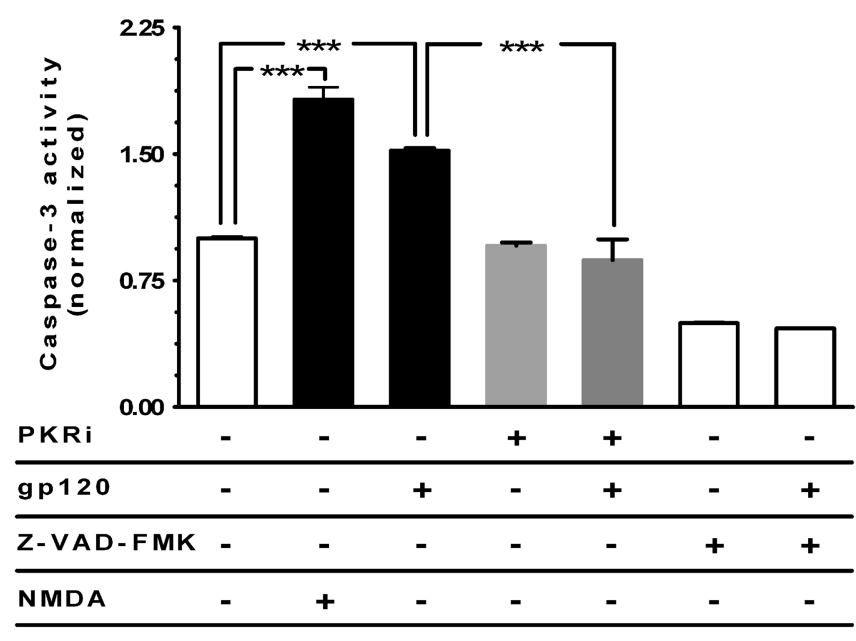

Figure 4. PKR inactivation inhibits caspase-3 activity induced by gp120. Cultures were pretreated with $200 \mathrm{~nm}$ PKRi and then exposed to gp120 for $18 \mathrm{~h}$. PKRi pretreatment in cortical cultures reduced caspase-3 activity induced by gp120. NMDA (100 $\mu \mathrm{m}$ for $10 \mathrm{~min}$ ) was used as a positive control for activating caspase-3. Z-VAD-FMK ( $N$-benzyloxycarbonyl-Val-Ala-Aspfluoromethylketone) (an irreversible inhibitor of caspase-3 activity) was used as a negative control for the assay. ${ }^{* *} p<0.001$ for $n=4$ experiments. Values are mean \pm SEM fold increase in activity.

patients with HAD, suggesting the pathophysiological relevance of this new cell death pathway.

Until recently, the only known PKR inducers were lipopolysaccharide, serum starvation, or cytokines, such as IFN- $\alpha,-\beta$, and $-\gamma$, tumor necrosis factor- $\alpha$, and interleukin-1 $\beta$ (Peel, 2004;
Takada et al., 2007). Here, we report gp120 as a new inducer of PKR activity that contributes to neuronal apoptosis. Our results show that PKR was induced almost exclusively in neurons and only to a minor degree in a small number of astrocytes. PKR upregulation by itself can potentiate its own activation (Bonnet et al., 2000) and promote apoptosis. We found that, after gp120 exposure, the PKR activation in neurons contributes to the downstream activation of a caspase-3-dependent apoptotic signaling cascade. In addition to PKR upregulation by gp120, the presence of PACT in neurons may potentiate PKR activation and signaling in neuronal apoptosis. PACT is known to mediate stress-induced apoptosis and therefore may amplify neuronal apoptosis under our conditions. To identify the mechanism of PACT activity in gp120-induced neurodegeneration, additional research needs to examine the neurotoxicity induced by gp120 on cultures derived from PACT and double PACT/PKR knock-out mice.

We also sought to determine whether the upregulation and activation of PKR contributed to neuronal apoptosis. We therefore used two pharmacological inhibitors, 2-AP and PKRi, and found that both abrogated neuronal apoptosis induced by gp 120 . We further validated this hypothesis by showing that gp120 neurotoxicity was essentially abrogated in cells genetically deficient in PKR. Although other studies are needed to assess whether gp120 may be working through chemokine receptors, analysis of both brain tissue as well as the mixed cultures derived from PKRdeficient mice revealed no change in CXCR4 or CCR5 expression levels (our unpublished data).

Thus, activation of PKR appears to exert a central function in gp120-associated neurodegeneration in mixed cortical cell cul- 

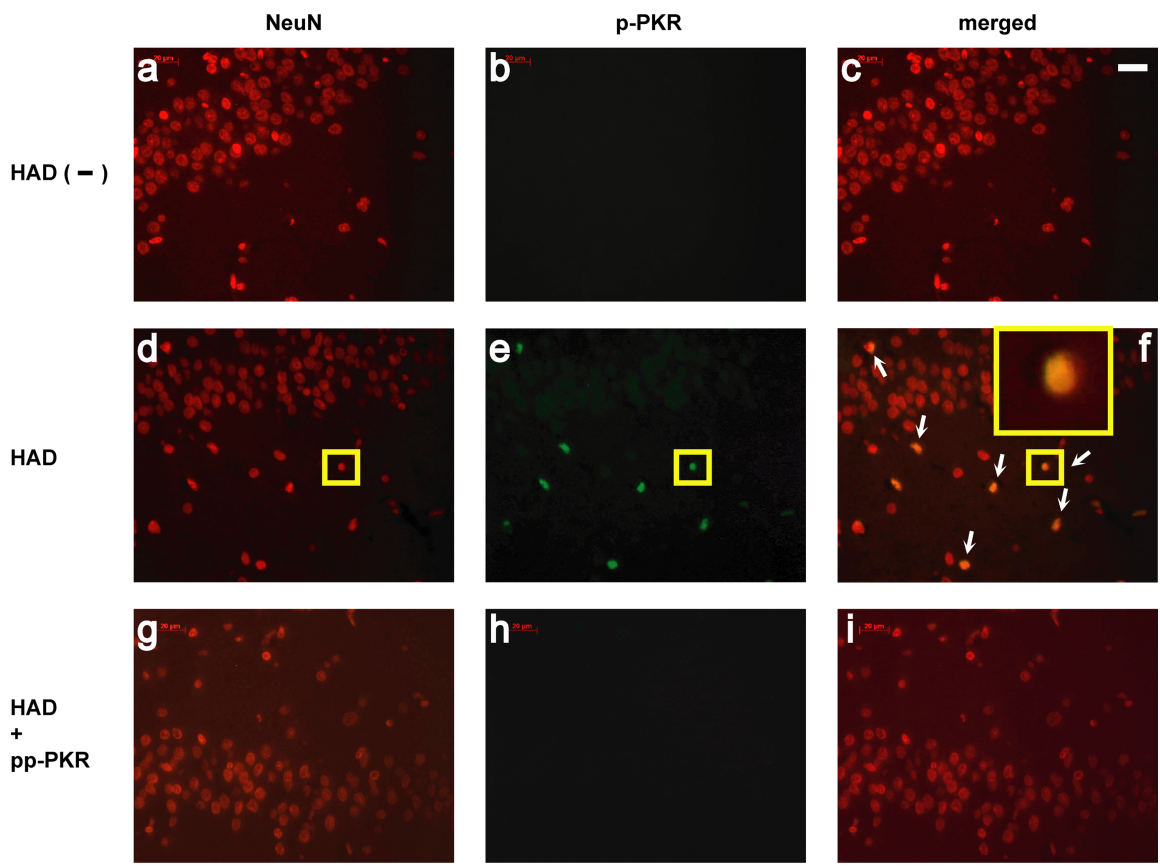

Figure 5. The activated form of PKRi localized in neuronal nuclei from autopsied brain sections of HAD patients. $\boldsymbol{a}-\boldsymbol{c}$, Hippocampal tissue from an HIV-positive but HAD-negative brain that had been double labeled with anti-NeuN (red) and anti-p-PKR (green) but no p-PKR labeling. $\boldsymbol{d}-\boldsymbol{f}$, Hippocampal tissue from an HAD patient manifested numerous NeuN-labeled cells colocalized with p-PKR (white arrows). In $\boldsymbol{f}$, a magnification of the immunofluorescent image of one cell (small yellow box, $\boldsymbol{d}-\boldsymbol{f}$ ) is shown in the inset. $\boldsymbol{g}-\boldsymbol{i}$, Absence of p-PKR staining on HAD sections after incubation with pp-PKR. Results represent findings in brain sections from five HAD-positive individuals and five control patients including HIV infection but not HAD. Scale bar, $20 \mu \mathrm{m}$.
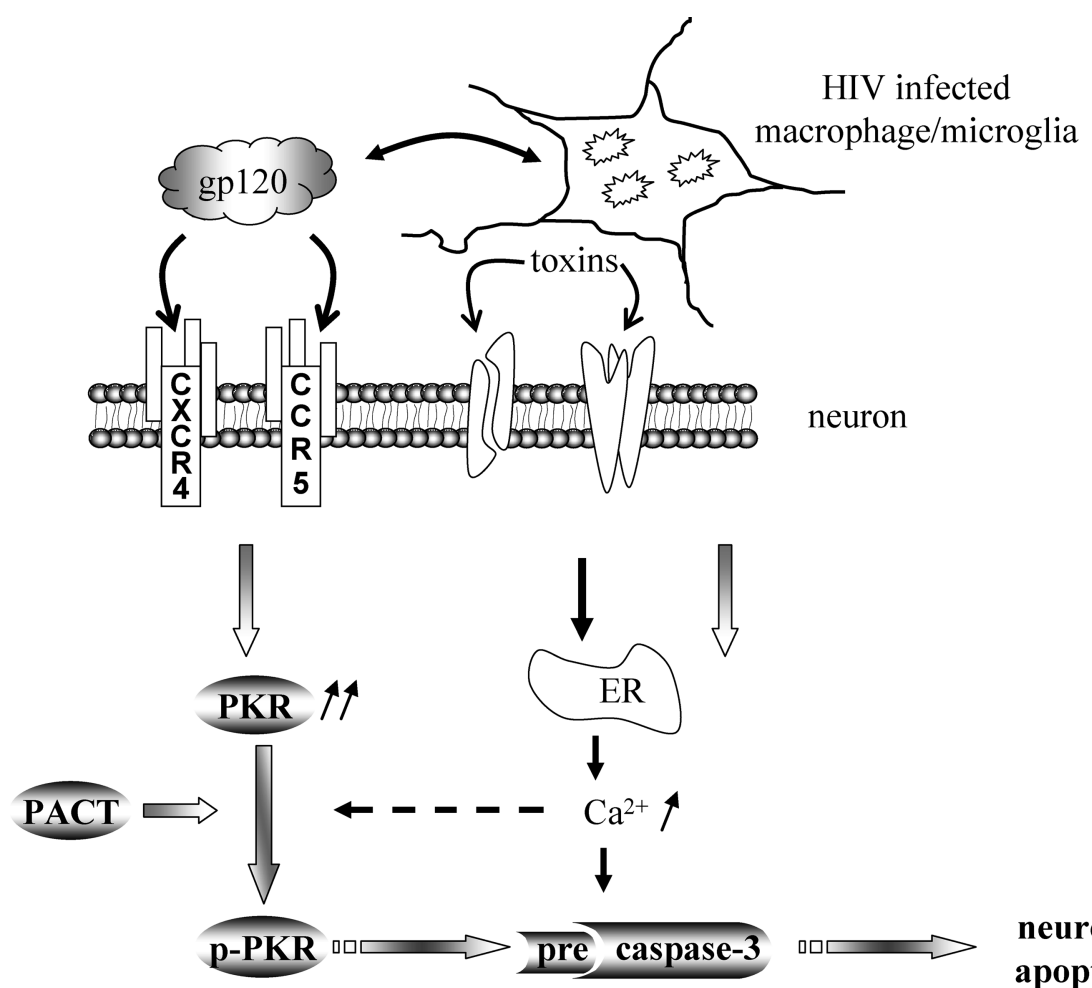

neuronal apoptosis

Figure 6. Model of PKR activation in HIV-1/gp120 induced-neuronal apoptosis. Direct or indirect effects of gp120 may mediate PKR induction in neurons, in which the presence of PACT can lead to activation PKR in the absence of dsRNA. Phospho/activatedPKR induces caspase-3 activation, which mediates neuronal apoptosis. gp120 can activate chemokine receptors that function as CD4 coreceptors (such as CXCR4 and CCR5) to evoke $\mathrm{Ca}^{2+}$ release from ER stores and may activate PKR. Caspase-3 activation can also be induced by gp 120 through a mechanism independent of PKR activation, such as ER stress resulting from toxins released by infected or immune-stimulated macrophage/microglia. tures. Additionally, we found that PKR activation was associated with its translocation to nucleus during gp120 treatment. Clearly, PKR and its activation are necessary for gp120 induction of neuronal apoptosis. Hence, activated PKR not only plays a role in neurodegeneration induced by gp120 but is also a potential target for therapeutic intervention against HAD. Additionally, we showed that caspase- 3 activation, known to occur during gp120induced neuronal apoptosis (Garden et al., 2002), was diminished by PKR inhibitors.

Importantly, the presence of phosphorylated/activated PKR in specimens of HAD postmortem brain, but not specimens from those with HIV but not dementia, supports the notion that HIV/gp120activated PKR may play a role in HAD pathogenesis. The presence of $\mathrm{p}-\mathrm{PKR}$ was shown previously in other neurodegenerative diseases (Peel et al., 2001; Peel and Bredesen, 2003; Onuki et al., 2004; Bando et al., 2005), and here we show for the first time a potential role in HIV-associated neurodegeneration. Notably, total PKR was localized throughout the cytosol of neurons that were not exposed to gp120, but nuclear localization of PKR, especially $\mathrm{p}-\mathrm{PKR}$, was greater in neurons exposed to gp120. Furthermore, analysis of human postmortem brain also indicated the presence of the activated form of PKR mostly in neuronal nuclei, suggesting that PKR might be translocated into these nuclei to target nuclear substrates such as the transcription factor p53. These findings are consistent with previous work that had shown that, although unphosphorylated PKR is more highly expressed in the cytosol than in the nucleus, during activation by dsRNA, phosphorylated PKR translocates to the nucleus (Takizawa et al., 2000). This would explain our results in which staining of phosphorylated PKR was greatest in the neuronal nuclei of brain samples from patients with HAD. However, the possibility remains that the staining was altered by postmortem events.

Our demonstration that gp120 activates the PKR signaling pathway adds to our knowledge regarding the mechanisms of HIV-induced neuropathogenesis. However, we do not known whether PKR is activated by a direct pathway in neurons or through an indirect pathway. Regardless, gp120 leads to PKR activation and subsequent caspase-3 activity in neurons (Fig. 6). It will be important for future studies to examine other PKR substrates that may potentially contribute to neuronal apoptosis, including eukaryotic initia- 
tion factor $2 \alpha$, E2 promoter binding factor-1 (E2F-1), p53, or p38 mitogen-activated protein kinase (MAPK) (Chang et al., 2002a,b; Suen et al., 2003; Garcia et al., 2006). Because gp120 is also known to activate p38 MAPK (Kaul and Lipton, 1999; Kaul et al., 2001, 2007) and transcription factors such as E2F-1 and p53 in neurons (Khan et al., 2003; Garden et al., 2004), it is possible that PKR plays a role in this activation. Moreover, inhibition of protein translation through PKR activation might have an impact on the de novo synthesis of proteins attributable to changes of protein patterns in the cells. For example, cycloheximide depresses overall protein synthesis, whereas it increases the synthesis of $\alpha \mathrm{Ca}^{2+}$ / calmodulin dependant kinase II at developing synaptic contacts (Scheetz et al., 2000).

In conclusion, the concordance between our results on cultured rodent cerebrocortical cells and autopsy material from humans with HAD suggests that PKR and downstream effectors may participate in the pathogenesis of HAD. Thus, this newly recognized pathway to neuronal damage and cell death may offer a novel target for therapeutic intervention in HAD. PACT/PKR interactions may be an important phase to consider in all HIVassociated neurodegeneration. Identifying the mechanisms of PKR activation by gp120 may yield important insights, hypotheses, and alternative drugs to control the initial triggers and early events that promote the progression of this devastating disease.

\section{References}

Bagetta G, Corasaniti MT, Aloe L, Berliocchi L, Costa N, Finazzi-Agro A, Nistico G (1996) Intracerebral injection of human immunodeficiency virus type 1 coat protein gp120 differentially affects the expression of nerve growth factor and nitric oxide synthase in the hippocampus of rat. Proc Natl Acad Sci USA 93:928-933.

Balachandran S, Kim CN, Yeh WC, Mak TW, Bhalla K, Barber GN (1998) Activation of the dsRNA-dependent protein kinase, PKR, induces apoptosis through FADD-mediated death signaling. EMBO J 17:6888-6902.

Bando Y, Onuki R, Katayama T, Manabe T, Kudo T, Taira K, Tohyama M (2005) Double-strand RNA dependent protein kinase (PKR) is involved in the extrastriatal degeneration in Parkinson's disease and Huntington's disease. Neurochem Int 46:11-18.

Bonnet MC, Weil R, Dam E, Hovanessian AG, Meurs EF (2000) PKR stimulates NF-kappaB irrespective of its kinase function by interacting with the IkappaB kinase complex. Mol Cell Biol 20:4532-4542.

Bonnet MC, Daurat C, Ottone C, Meurs EF (2006) The N-terminus of PKR is responsible for the activation of the NF-kappaB signaling pathway by interacting with the IKK complex. Cell Signal 18:1865-1875.

Brenneman DE, Westbrook GL, Fitzgerald SP, Ennist DL, Elkins KL, Ruff MR, Pert CB (1988) Neuronal cell killing by the envelope protein of HIV and its prevention by vasoactive intestinal peptide. Nature 335:639-642.

Chang RC, Wong AK, Ng HK, Hugon J (2002a) Phosphorylation of eukaryotic initiation factor-2alpha (eIF2alpha) is associated with neuronal degeneration in Alzheimer's disease. NeuroReport 13:2429-2432.

Chang RC, Suen KC, Ma CH, Elyaman W, Ng HK, Hugon J (2002b) Involvement of double-stranded RNA-dependent protein kinase and phosphorylation of eukaryotic initiation factor-2alpha in neuronal degeneration. J Neurochem 83:1215-1225.

Dreyer EB, Kaiser PK, Offermann JT, Lipton SA (1990) HIV-1 coat protein neurotoxicity prevented by calcium channel antagonists. Science 248:364-367.

Garcia MA, Gil J, Ventoso I, Guerra S, Domingo E, Rivas C, Esteban M (2006) Impact of protein kinase PKR in cell biology: from antiviral to antiproliferative action. Microbiol Mol Biol Rev 70:1032-1060.

Garden GA, Budd SL, Tsai E, Hanson L, Kaul M, D’Emilia DM, Friedlander RM, Yuan J, Masliah E, Lipton SA (2002) Caspase cascades in human immunodeficiency virus-associated neurodegeneration. J Neurosci 22:4015-4024.

Garden GA, Guo W, Jayadev S, Tun C, Balcaitis S, Choi J, Montine TJ, Moller T, Morrison RS (2004) HIV associated neurodegeneration requires p53 in neurons and microglia. FASEB J 18:1141-1143.

Gilbert SJ, Duance VC, Mason DJ (2002) Tumour necrosis factor alpha upregulates protein kinase $\mathrm{R}$ (PKR)-activating protein (PACT) and in- creases phosphorylation of PKR and eukaryotic initiation factor 2-alpha in articular chondrocytes. Biochem Soc Trans 30:886-889.

Haughey NJ, Mattson MP (2002) Calcium dysregulation and neuronal apoptosis by the HIV-1 proteins Tat and gp120. J Acquir Immune Defic Syndr 31 [Suppl 2]:S55-S61.

Hu JH, Zhang H, Wagey R, Krieger C, Pelech SL (2003) Protein kinase and protein phosphatase expression in amyotrophic lateral sclerosis spinal cord. J Neurochem 85:432-442.

Huang S, Qu LK, Cuddihy AR, Ragheb R, Taya Y, Koromilas AE (2003) Protein kinase inhibitor 2-aminopurine overrides multiple genotoxic stress-induced cellular pathways to promote cell survival. Oncogene 22:3721-3733.

Iwamura T, Yoneyama M, Koizumi N, Okabe Y, Namiki H, Samuel CE, Fujita $\mathrm{T}$ (2001) PACT, a double-stranded RNA binding protein acts as a positive regulator for type I interferon gene induced by Newcastle disease virus. Biochem Biophys Res Commun 282:515-523.

Jagus R, Joshi B, Barber GN (1999) PKR, apoptosis and cancer. Int J Biochem Cell Biol 31:123-138.

Jammi NV, Whitby LR, Beal PA (2003) Small molecule inhibitors of the RNA-dependent protein kinase. Biochem Biophys Res Commun 308:50-57.

Kaul M, Lipton SA (1999) Chemokines and activated macrophages in HIV gp120-induced neuronal apoptosis. Proc Natl Acad Sci USA 96:8212-8216.

Kaul M, Garden GA, Lipton SA (2001) Pathways to neuronal injury and apoptosis in HIV-associated dementia. Nature 410:988-994.

Kaul M, Ma Q, Medders KE, Desai MK, Lipton SA (2007) HIV-1 coreceptors CCR5 and CXCR4 both mediate neuronal cell death but CCR5 paradoxically can also contribute to protection. Cell Death Differ 14:296-305.

Khan MZ, Brandimarti R, Musser BJ, Resue DM, Fatatis A, Meucci O (2003) The chemokine receptor CXCR4 regulates cell-cycle proteins in neurons. J Neurovirol 9:300-314.

Lannuzel A, Lledo PM, Lamghitnia HO, Vincent JD, Tardieu M (1995) HIV-1 envelope proteins gp120 and gp160 potentiate NMDA-induced $\left[\mathrm{Ca}^{2+}\right] \mathrm{i}$ increase, alter $\left[\mathrm{Ca}^{2+}\right] \mathrm{i}$ homeostasis and induce neurotoxicity in human embryonic neurons. Eur J Neurosci 7:2285-2293.

Lei SZ, Zhang D, Abele AE, Lipton SA (1992) Blockade of NMDA receptormediated mobilization of intracellular $\mathrm{Ca}^{2+}$ prevents neurotoxicity. Brain Res 598:196-202.

Lipton SA, Gendelman HE (1995) Seminars in medicine of the Beth Israel Hospital, Boston. Dementia associated with the acquired immunodeficiency syndrome. N Engl J Med 332:934-940.

Lipton SA, Sucher NJ, Kaiser PK, Dreyer EB (1991) Synergistic effects of HIV coat protein and NMDA receptor-mediated neurotoxicity. Neuron 7:111-118.

Mattson MP, Haughey NJ, Nath A (2005) Cell death in HIV dementia. Cell Death Differ 12 [Suppl 1]:893-904.

McArthur JC, Brew BJ, Nath A (2005) Neurological complications of HIV infection. Lancet Neurol 4:543-555.

Medina I, Ghose S, Ben-Ari Y (1999) Mobilization of intracellular calcium stores participates in the rise of $\left[\mathrm{Ca}^{2+}\right] \mathrm{i}$ and the toxic actions of the HIV coat protein gp120. Eur J Neurosci 11:1167-1178.

Meucci O, Fatatis A, Simen AA, Bushell TJ, Gray PW, Miller RJ (1998) Chemokines regulate hippocampal neuronal signaling and gp120 neurotoxicity. Proc Natl Acad Sci USA 95:14500-14505.

Onuki R, Bando Y, Suyama E, Katayama T, Kawasaki H, Baba T, Tohyama M, Taira K (2004) An RNA-dependent protein kinase is involved in tunicamycin-induced apoptosis and Alzheimer's disease. EMBO J 23:959-968.

Patel RC, Sen GC (1998) PACT, a protein activator of the interferoninduced protein kinase, PKR. EMBO J 17:4379-4390.

Peel AL (2004) PKR activation in neurodegenerative disease. J Neuropathol Exp Neurol 63:97-105.

Peel AL, Bredesen DE (2003) Activation of the cell stress kinase PKR in Alzheimer's disease and human amyloid precursor protein transgenic mice. Neurobiol Dis 14:52-62.

Peel AL, Rao RV, Cottrell BA, Hayden MR, Ellerby LM, Bredesen DE (2001) Double-stranded RNA-dependent protein kinase, PKR, binds preferentially to Huntington's disease (HD) transcripts and is activated in HD tissue. Hum Mol Genet 10:1531-1538.

Peters GA, Hartmann R, Qin J, Sen GC (2001) Modular structure of PACT: 
distinct domains for binding and activating PKR. Mol Cell Biol 21:1908-1920.

Prostko CR, Dholakia JN, Brostrom MA, Brostrom CO (1995) Activation of the double-stranded RNA-regulated protein kinase by depletion of endoplasmic reticular calcium stores. J Biol Chem 270:6211-6215.

Scheetz AJ, Nairn AC, Constantine-Paton M (2000) NMDA receptormediated control of protein synthesis at developing synapses. Nat Neurosci 3:211-216.

Scheuner D, Patel R, Wang F, Lee K, Kumar K, Wu J, Nilsson A, Karin M, Kaufman RJ (2006) Double-stranded RNA-dependent protein kinase phosphorylation of the alpha-subunit of eukaryotic translation initiation factor 2 mediates apoptosis. J Biol Chem 281:21458-21468.

Shimazawa M, Hara H (2006) Inhibitor of double stranded RNAdependent protein kinase protects against cell damage induced by ER stress. Neurosci Lett 409:192-195.

Srivastava SP, Kumar KU, Kaufman RJ (1998) Phosphorylation of eukaryotic translation initiation factor 2 mediates apoptosis in response to activation of the double-stranded RNA-dependent protein kinase. J Biol Chem 273:2416-2423.

Suen KC, Yu MS, So KF, Chang RC, Hugon J (2003) Upstream signaling pathways leading to the activation of double-stranded RNA-dependent serine/threonine protein kinase in beta-amyloid peptide neurotoxicity. J Biol Chem 278:49819-49827.
Takada Y, Ichikawa H, Pataer A, Swisher S, Aggarwal BB (2007) Genetic deletion of PKR abrogates TNF-induced activation of IkappaBalpha kinase, JNK, Akt and cell proliferation but potentiates p44/p42 MAPK and p38 MAPK activation. Oncogene 26:1201-1212.

Takizawa T, Tatematsu C, Watanabe M, Yoshida M, Nakajima K (2000) Three leucine-rich sequences and the N-terminal region of doublestranded RNA-activated protein kinase (PKR) are responsible for its cytoplasmic localization. J Biochem (Tokyo) 128:471-476.

Toggas SM, Masliah E, Rockenstein EM, Rall GF, Abraham CR, Mucke L (1994) Central nervous system damage produced by expression of the HIV-1 coat protein gp120 in transgenic mice. Nature 367:188-193.

Uehara T, Nakamura T, Yao D, Shi ZQ, Gu Z, Ma Y, Masliah E, Nomura Y, Lipton SA (2006) S-nitrosylated protein-disulphide isomerase links protein misfolding to neurodegeneration. Nature 441:513-517.

Williams BR (1999) PKR; a sentinel kinase for cellular stress. Oncogene 18:6112-6120.

Yang YL, Reis LF, Pavlovic J, Aguzzi A, Schafer R, Kumar A, Williams BR, Aguet M, Weissmann C (1995) Deficient signaling in mice devoid of double-stranded RNA-dependent protein kinase. EMBO J 14:6095-6106.

Yeung MC, Liu J, Lau AS (1996) An essential role for the interferoninducible, double-stranded RNA-activated protein kinase PKR in the tumor necrosis factor-induced apoptosis in U937 cells. Proc Natl Acad Sci USA 93:12451-12455. 